\title{
Investigation on interdigitated electrode design for impedance spectroscopy technique targeting lard detection application
}

\begin{abstract}
This paper investigates the interdigitated electrode design for impedance spectroscopy technique targeting lard detection purposes. Two types of interdigitated electrode were designed and evaluated on high precision impedance converter board subjected to frequency range of $5 \mathrm{kHz}$ to $100 \mathrm{kHz}$. Samples of lard and beef tallow were extracted and mixed with hexane solvent for different concentration levels. Analysis of variance (ANOVA) was applied to the experimental data for statistical data analysis. Experimental results indicate that the impedance value is not a strong function of concentration levels but a function of frequencies. ANOVA results have confirmed that IDE Type-B that has larger electrode area has higher sensing sensitivity over Type-A. Principal Component Analysis (PCA) was used as a statistical multivariate modeling. PCA analysis results showed that the IDE Type B could group animal fats into two distinct groups using both parameters of frequencies and concentrations.
\end{abstract}

Keyword: Interdigitated electrode design; Impedance spectroscopy; Printed circuit board (PCB); Lard detection; Analysis of variance (ANOVA); Principal component analysis (PCA) 